\title{
HEART FAILURE WITH NORMAL AND REDUCED EJECTION FRACTION - ASSESSMENT AND SHARED CARE MANAGEMENT
}

Dr Rohit Khurana

\section{INTRODUCTION}

Heart failure (HF) is a common clinical syndrome resulting from any structural or functional cardiac disorder that impairs the ability of the ventricle to fill with or eject blood. HF may be caused by disease of the myocardium, pericardium, endocardium, heart valves, vessels, or by metabolic disorders. HF due to left ventricular dysfunction is categorized into HF with reduced ejection fraction (with Left Ventricular Ejection Fraction (LVEF) $\leq \mathbf{5 0}$ percent, known as HFrEF; also referred to as systolic HF) and HF with preserved ejection fraction (with LVEF > 50 percent; known as HFpEF; also referred to as diastolic HF. ${ }^{1}$ A reduced LVEF in systolic heart failure is a powerful predictor of mortality. As many as $40-50$ percent of patients with heart failure have diastolic heart failure with preserved left ventricular function. Overall, there is no difference in survival between diastolic and systolic heart failure that cannot be attributed to ejection fraction. Patients with diastolic heart failure are more likely to be women, to be older, and to have hypertension, atrial fibrillation, and left ventricular hypertrophy, but no history of coronary artery disease. ${ }^{2,3}$ The pathogenesis of diastolic dysfunction involves abnormalities of active ventricular relaxation and passive ventricular compliance, which lead to ventricular stiffness and higher diastolic pressures. These pressures are transmitted through atrial and pulmonary venous systems, reducing lung compliance. A combination of decreased lung compliance and cardiac output leads to symptoms. ${ }^{2}$

Keywords: Heart Failure, shared care management, reduced ejection fraction, preserved ejection

fraction, BNP, therapy

SFP2019; 45(2) : 27-31

\section{Clinical presentation and diagnosis}

Patients with HF usually present with the classic triad of symptoms - oedema, fatigue, and dyspnea. Other typical symptoms may include orthopnea, paroxysmal nocturnal dyspnea, reduced exercise tolerance, and increased time to recover from exercise. Less typical signs include nocturnal cough, wheezing, bloated feeling, loss of appetite, confusion (especially in the elderly), depression, palpitations, dizziness and syncope. ${ }^{4}$ However,

\section{DR ROHIT KHURANA}

Consultant Senior Consultant, General and Interventional Cardiologist

The Harley Street Heart and Vascular Centre

Gleneagles Hospital symptoms are non-specific and non-sensitive, and therefore are less useful in discriminating HFrEF from HFpEF. In addition, atypical presentations should be considered when evaluating obese patients and older adults because of potentially different aetiology, clinical presentation and outcome as compared to the general population. Typical signs of HFrEF include elevatedjugular venous pressure, gallop rhythm, hepato-jugular reflux, and laterally displaced apical impulse. Less typical symptoms include weight gain, cachexia (tissue wasting), cardiac murmur, peripheral edema (ankle, sacral, scrotal), pulmonary rales, tachycardia, irregular pulse, tachypnea, hepatomegaly, ascites, cold extremities, oliguria and narrow pulse pressure. ${ }^{4}$ The assessment of signs and symptoms is clinically significant to suggest the likelihood of HFrEF as well as to monitor response to therapy and stability overtime. In groups such as the obese, the elderly and those with chronic lung disease, being confident of the symptoms and signs may be more difficult.

Persistent symptoms despite treatment often suggest the need for additional therapy and worsening of symptoms often suggest serious development and the need for prompt medical attention.

Heart failure is much more likely in the presence of a relevant medical history suggesting an increased risk of cardiac damage. In advanced economies such as Singapore, nearly 60 percent of patients diagnosed with acute heart failure have underlying coronary artery disease and in patients with acute coronary syndromes, myocardial ischaemia is often a precipitant risk factor, especially for de novo heart failure. ${ }^{5}$ A prior diagnosis of heart failure, diabetes, hypertension, valvular heart disease, advanced age, male gender and obesity have all been found to predict fluid overload typical of congestive heart failure.

The symptoms and signs of heart failure are the consequence of systemic and pulmonary congestion which result from increased left ventricular filling pressures. Even in the absence of overt clinical congestion, hemodynamic congestion may still occur and this also predicts a worse mortality and re-hospitalization rate. Heart failure symptoms can occur with preserved or reduced ejection fraction, (systolic or diastolic heart failure). The New York Heart Association classification system is the simplest and most widely used method to gauge symptom severity (Table 1). The classification system is a well-established predictor of mortality and can be used at diagnosis and to monitor treatment response. 


\section{Assessment}

In patients with non-acute onset, presenting with symptoms and signs of heart failure in the primary care setting, the probability of heart failure should be determined by the history and symptoms supported by findings on clinical examination. Blood tests, an ECG and a chest $\mathrm{x}$-ray should be requested on all patients. During these steps, at least one element should be positive to consider the diagnosis and then plasma natriuretic peptides and an echocardiogram should be requested. The diagnostic assessment, in accordance with the ESC 2016 guidelines is summarized in Table 2.4

\section{Brain Natriuretic Peptide. (BNP)}

BNP and N-terminal pro-BNP (the cleaved inactive N-terminal fragment of the BNP precursor) levels can be used to evaluate patients with shortness of breath suspected of having heart failure. ${ }^{5} \mathrm{BNP}$ is secreted by the atria and ventricles in response to stretching or increased wall tension. The hormone then causes fluid and sodium loss in the urine and mild vasodilation. BNP levels increase with age, is higher in women and patients of African origin, and can be elevated in patients with renal failure. $\mathrm{BNP}$ appears to have better reliability than $\mathrm{N}$-terminal pro- $\mathrm{BNP}^{6}$, especially in older populations. Guidelines from the American College of Cardiology/American Heart Association (ACC/ AHA) and European Society of Cardiology (ESC) recommend the use of natriuretic peptides for assessment of patients with symptoms of heart failure.

Most dyspneic patients with $\mathrm{HF}$ have plasma BNP values > $400 \mathrm{pg} / \mathrm{mL}$, while values $<100 \mathrm{pg} / \mathrm{mL}$ have a very high negative predictive value for $\mathrm{HF}$ as a cause of dyspnea. ${ }^{7}$ In the range between 100-400 pg/mL, plasma BNP concentrations are not very sensitive or specific for detecting or excluding HF. As BNP levels increase, the specificity increases and thus the likelihood of a heart failure diagnosis. BNP levels also increase in level according to New York Heart Association classification. Elevations in plasma BNP can establish the presence of HF due to diastolic dysfunction with similar accuracy to systolic dysfunction. However, the values do not differentiate between systolic and diastolic dysfunction.

BNP levels are strong predictors of mortality at two to three months and cardiovascular events in acute heart failure, specifically when BNP level is > $200 \mathrm{pg} / \mathrm{mL}$ or N-terminal pro-BNP level is $>5,180 \mathrm{pg} / \mathrm{mL}^{7}{ }^{7}$ Limited evidence supports monitoring reduction of BNP levels in the acute and outpatient settings. A 30 to 50 percent reduction in BNP level at hospital discharge showed improved survival and reduced rehospitalization rates. Optimizing management for outpatient targets of a BNP level $<100 \mathrm{pg} / \mathrm{mL}$ and an N-terminal pro-BNP level $<1,700 \mathrm{pg} / \mathrm{mL}$ showed improvement in decompensations, hospitalizations, and mortality events. ${ }^{7}$

\section{Echocardiography}

Echocardiography should be performed in all patients with new onset $\mathrm{HF}$ and can provide important information about ventricular size and function. ${ }^{8}$ The sensitivity and specificity of two-dimensional echocardiography for the diagnosis of systolic dysfunction are as high as 80 and 100 percent, respectively. Valvular structure and function in valve disease can be characterized. A number of other important findings can be detected. Regional wall motion abnormalities are compatible with coronary artery disease. Pericardial thickening may be indicative of constrictive pericarditis. Infiltrative cardiomyopathies are associated with an abnormal myocardial texture. Left ventricular diastolic function can be assessed by estimation of the pulmonary capillary wedge pressure via the ratio of tissue Doppler of early mitral inflow velocity (E) to early diastolic velocity of the mitral annulus (e'). An E/e' ratio $>15$ suggests a Pulmonary capillary wedge pressure (PCWP) $>15 \mathrm{mmHg}$ when $\mathrm{e}^{\prime}$ is the mean of medial and lateral mitral annulus early diastolic velocities. There are limitations to use of the E/e' ratio, which are beyond the scope of this article. Right atrial and pulmonary artery pressures, determined by the peak velocity of tricuspid regurgitation on Doppler echocardiography. These findings correlate with the pulmonary artery wedge pressure, regardless of the etiology of HF or severity of tricuspid regurgitation; they can be used to assess changes in left ventricular filling pressures resulting from therapy.

\section{Treadmill Exercise Testing}

Exercise testing should be part of the initial evaluation of virtually all patients with $\mathrm{HF}$. In addition to detection of ischemic heart disease, assessment of exercise capacity can be used for risk stratification and determining prognosis; serial measurements also can assess the efficacy of therapy and clinical stability of patients over time.

With severe HF, measurement of the maximal oxygen uptake (VO2max) provides an objective estimate of the functional severity of the myocardial dysfunction. ${ }^{9} \mathrm{VO} 2 \mathrm{max}$ is one of the best indices of prognosis in patients with symptomatic HF. However, peak VO2 and exercise capacity can be affected by factors other than cardiac status, including deconditioning, pulmonary disease, and anemia. One advantage of measuring VO2max directly is that cardiac and non-cardiac causes of impaired exercise can be distinguished by assessing the anaerobic threshold and related indices.

\section{Principles of Management}

The 2016 ESC guidelines recommend both lifestyle interventions and pharmacological therapies. ${ }^{4} \mathrm{HF}$ with reduced ejection fraction has well-validated therapies to reduce morbidity (i.e., reducing symptoms, improving health-related quality of life and functional status, decreasing the rate of hospitalization), and to reduce mortality. In contrast, $\mathrm{HF}$ with preserved ejection fraction lacks evidence-based treatment recommendations. Diuretics are essential in relieving symptoms but it has yet to be definitively established whether they provide long term prognostic benefit. ${ }^{10}$ They provide immediate relief of symptoms and help manage the chronic fluid status in stable patients, irrespective of whether it is HFrEF or HFpEF. It is imperative that patients with heart failure understand their condition and are involved in their management decisions; lifestyle interventions can improve patients' quality of life and prevent exacerbations. The role of dietary salt restriction 
and the importance of regular exercise to increase functional capacity should be reinforced. For patients with more advanced heart failure, daily weight measurements and fluid restriction with more close supervision may be necessary. Formalized cardiac rehabilitation which combines exercise with ongoing educational and psychological support has proven benefit.

\section{HF with reduced ejection fraction}

Management of heart failure with reduced ejection fraction (HFrEF) includes management of contributing factors and associated conditions, lifestyle modification, pharmacologic therapy and if indicated, device therapy(Figure 1).Cardiac resynchronization therapy is indicated with patients with an ejection fraction $<35$ percent and QRS duration of $>130 \mathrm{~ms}$. Contributing factors comprise hypertension, myocardial ischemia or infarction, diabetes mellitus, thyroid dysfunction, and infection. Coronary disease and diabetes mellitus have become increasingly responsible for $\mathrm{HF}$ while hypertension and valve disease have become less common because of improvements in detection and therapy. However, almost all patients with HFrEF will require diuretics on a chronic basis to alleviate symptoms and signs of congestion. ${ }^{11}$ Other treatment generally starts with Ace inhibitors or angiotensin receptor blockers (ARBs), followed by beta-blockers. Beta blockers can also provide relief in patients with ischemic heart disease presenting with angina and rate control in those with atrial fibrillation. If symptoms persist, as based on the NYHA functional classification of II-IV, mineralocorticoid receptor antagonists (MRA such as spironolactone or eplerenone may be added. Ivabradine may also be an option for some patients with HFrEF, if they are in sinus rhythm and their resting heart rate > $70 \mathrm{bpm}$. Improved mortality has been demonstrated with beta blockers, ACE inhibitors, ARNI, hydralazine plus nitrate, and MRAs, with limited evidence of survival benefit for diuretics.

\section{Novel Treatment options in HFrEF}

One new treatment strategy is the angiotensin receptor neprilysin inhibitor (ARNI), which comprises valsartan and sacubitril. This drug serves to reduce sympathetic tone, aldosterone levels and sodium retention through inhibition of the over active renin angiotensin system while simultaneously potentiating protective vasoreactive neuropeptides. The first (and currently only commercially available) ARNI, was formerly known as LCZ696 and marketed as Entresto, was evaluated in the PARADIGM$\mathrm{HF}$ trial in comparison to enalapril 10mg bd. After 27 months of follow up, the trial was stopped early due to positive interim results. ${ }^{12}$ All-cause mortality was 17 percent with the ARNI, as compared to 19.8 percent with enalapril, in HFrEF patients maintained on optimal background HF therapy. This yielded a hazard ratio of $0.84(\mathrm{p}<0.001)$ and a number needed to treat of 32.The position of ARNI prescribing in primary care has also not been firmly established. Cardioselective beta-blockers may be prescribed in patients with co-morbid chronic obstructive pulmonary disease (COPD). More recently, the EMPA-REG OUTCOME and the DECLARE TIMI-58 trial reported that empagliflozin and dapagliflozin, respectively, which are both inhibitors of the sodium glucose co-transporter (SGLT-2) in the kidney when added to metformin, had beneficial prognostic cardiovascular outcomes compared to placebo in patients with cardiovascular risk factors and / or disease. ${ }^{12}$ Subgroup analysis suggested the benefits were consistent for patients with and without HF.

\section{HF with preserved ejection fraction}

In contrast with treatment of heart failure with HFrEF there are fewer randomized controlled trials (RCTs) of patients with HFpEF. The ACC/AHA recommends using a stage-based approach to guide treatment of HFpEF. ${ }^{13}$ The ESC recommends diuretics for treating fluid overload in patients with HFpEF. ${ }^{4}$ However, it makes no recommendation regarding other medications for treatment. It recommends identifying and treating cardiovascular and non-cardiovascular comorbidities, because most deaths and hospitalizations in patients with HFpEF are not due to chronic heart failure. The ACC/AHA and ESC recommend combined endurance and resistance training for patients with HFpEF to improve exercise capacity, physical functioning, and diastolic function.

Hypertension should be treated according to appropriate guidelines. Although RCTs of several medications showed fewer heart failure hospitalizations, this benefit was offset by increases in hospitalizations for other reasons. Thus, in the absence of hypertension, the evidence does not support treating patients with HFpEF with any medication except diuretics. Additionally, RCTs of angiotensin receptor blockers, nitrates, and spironolactone raise concerns about adverse effects, and physicians should avoid using these medications, if possible. Similarly, the use of digoxin should be avoided in patients 65 years and older. Comorbid atrial fibrillation or coronary artery disease should be treated.

Data from the PARAMOUNT trial comparing valsartan-sacubitril with valsartan in HFpEF showed that ARNI reduced NT-proBNP levels, left atrial volume index and increased eGFR more so than valsartan alone and independent of its systolic blood pressure lowering effect. The long-term clinical outcomes of ARNI in HFpEF are being further investigated in the ongoing PARAGON trial.

\section{Shared Care Management}

Often patients with HFrEF are managed in the hospital outpatient or specialist clinics for three to six months after diagnosis, to titrate medication to optimal doses. The optimal duration of these hospital-based programs before transitioning patient care to the community has not been established, nor whether all or only higher risk patients benefit. However, there is evidence that, given the complexity of the HF syndrome and its co-morbidities, close collaboration between hospital and primary care practitioners is crucial in order to provide optimal, integrated care and improved outcomes. ${ }^{14}$ It is important to realize that there is a wide variation in the disease trajectory of $\mathrm{HF}$ and the majority of patients do not generally follow a gradual decline. Some feel and function well then succumb to sudden fatality, while other patients may display improved functioning after a period of poor quality of life. Diverse and multiple comorbidities further complicate the disease trajectory warranting regular monitoring. 
Table 1: American College of Cardiology/American Heart Association Recommendations by Stage of Heart Failure ${ }^{13}$

American College of Cardiology/American Heart Association

Recommendations by Stage of Heart Failure

STAGE RECOMMENDATION

A: Heart failure risk factors

Guideline-directed treatment of hypertension and hyperlipidemia

B: Diastolic dysfunction without symptoms

Treat hypertension with thiazide diuretics, ACE inhibitors, or nondihydropyridine calcium channet blockers

C: Symptomatic heart failure with preserved ejection fraction and hypertension

Treat volume overload with diuretics; consider use of beta blockers, ACE inhibitors

Treat volume overload with diuretics

C: Symptomatic heart failure with preserved ejection fraction without hypertension

$A C E=$ angiotensin-converting enzyme; $A R B=$ angiotensin receptor blocker

Table 2: Diagnostic assessment in patient with suspected heart failure (non-acute onset) - according to ESC guidelines $^{4}$

Diagnostic assessment in patient with acute heart failure adapted from the ESC guidelines (2016) ${ }^{1}$

1. Measurement of plasma natriuretic peptide level (BNP, NT-proBNP or MR-proANP)*

2.

a) 12-lead ECG;

b) chest X-rayt

c) laboratory assessments: cardiac troponins, BUN (or urea), creatinine, electrolytes (sodium, potassium), glucose, complete blood count, liver function tests and TSH.

\section{Echocardiography¥}

* to help in the differentiation of acute heart failure from non-cardiac causes of acute dyspnea

$\dagger$ to assess signs of pulmonary congestion and detect other cardiac or non-cardiac diseases that may cause or contribute to the patient's symptoms

‡ recommended immediately in haemodynamically unstable patients with acute heart failure and within 48 hours when cardiac structure and function are either not known or may have changed since previous studies.
Figure 1. Therapeutic algorithm for a patient with symptomatic heart failure with reduced ejection fraction ${ }^{4}$

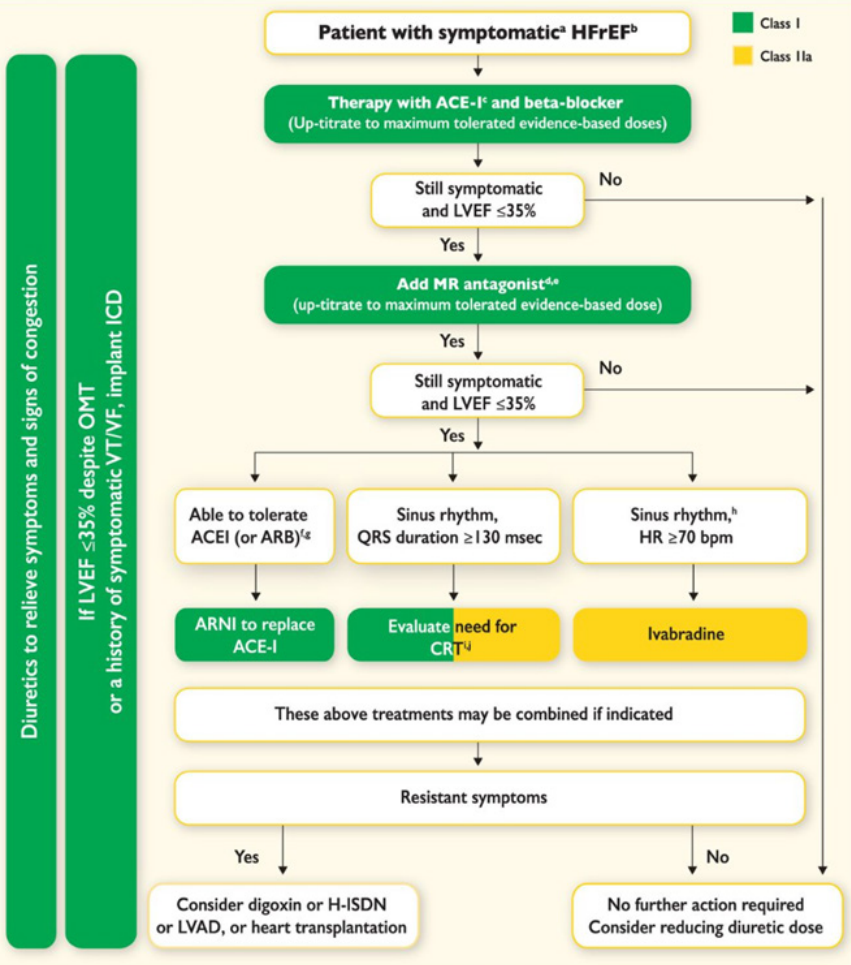




\section{REFERENCES}

1. Authors/Task Force Members, McMurray JJ, Adamopoulos S, Anker SD, Auricchio A Böhm M, Dickstein K, Falk V, Filippatos G, Fonseca C, Gomez-Sanchez MA. ESC Guidelines for the diagnosis and treatment of acute and chronic heart failure 2012: The Task Force for the Diagnosis and Treatment of Acute and Chronic Heart Failure 2012 of the European Society of Cardiology. Developed in collaboration with the Heart Failure Association (HFA) of the ESC. European Heart Journal. 2012 May 18; 33(14):1787-847.

2. Sharma K, Kass DA. Heart failure with preserved ejection fraction: mechanisms, clinical features, and therapies. Circulation research. 2014 Jun 20; 115(1):79-96.

3. Owan TE, Hodge DO, Herges RM, Jacobsen SJ, Roger VL, Redfield MM. Trends in prevalence and outcome of heart failure with preserved ejection fraction. New England Journal of Medicine. 2006 Jul 20; 355(3):251-9.

4. Ponikowski P, Voors AA, Anker SD, Bueno H, Cleland JG, Coats AJ, Falk V, Gonzálezjuanatey JR, Harjola VP, Jankowska EA, Jessup M. 2016 Esc Guidelines for the diagnosis and treatment of acute and chronic heart failure: The Task Force for the diagnosis and treatment of acute and chronic heart failure of the European Society of Cardiology (esc) developed with the special contribution of the Heart Failure Association (hfa) of the Esc. European Heart Journal. 2016 Jul 14; 37(27):2129-200.

5. Chen WC, Tran KD, Maisel AS. Biomarkers in heart failure. Heart. 2010 Feb 1; 96(4):314-20.

6. Kelder JC, Cramer MJ, van Wijngaarden J, van Tooren R, Mosterd A, Moons KG, Lammers JW, Cowie MR, Grobbee DE, Hoes AW. The diagnostic value of physical examination and additional testing in primary care patients with suspected heart failure. Circulation. 2011 Dec 20; 124(25):2865-73.

7. Maisel A, Mueller C, Adams Jr K, Anker SD, Aspromonte N, Cleland JG, Cohen-Solal A, Dahlstrom U, DeMaria A, Di Somma S, Filippatos GS. State of the art: using natriuretic peptide levels in clinical practice. European journal of heart failure. $2008 \mathrm{Sep}$; 10(9):824-39.
8. 2005 WRITING COMMITTEE MEMBERS, Hunt SA, Abraham WT, Chin MH, Feldman AM, Francis GS, Ganiats TG, Jessup M, Konstam MA, Mancini DM, Michl K. 2009 Focused Update Incorporated Into the ACC/AHA 2005 Guidelines for the Diagnosis and Management of Heart Failure in Adults: A Report of the American College of Cardiology Foundation/American Heart Association Task Force on Practice Guidelines: Developed in Collaboration With the International Society for Heart and Lung Transplantation. Circulation. 2009 Apr 14; 119(14):e391-479.

9. Ingle L. Prognostic value and diagnostic potential of cardiopulmonary exercise testing in patients with chronic heart failure. European journal of heart failure. $2008 \mathrm{Feb} ; 10(2): 112$ 8

10. von Lueder TG, Atar D, Krum H. Diuretic use in heart failure and outcomes. Clinical Pharmacology \& Therapeutics. 2013 Oct 1;94(4):490-8.

11. McMurray JJ, Packer M, Desai AS, Gong J, Lefkowitz MP, Rizkala AR, Rouleau JL, Shi VC, Solomon SD, Swedberg K, Zile MR. Angiotensin-neprilysin inhibition versus enalapril in heart failure. New England Journal of Medicine. 2014 Sep 11;371(11):993-1004.

12. Giugliano D, Meier JJ, Esposito K. Heart failure and type 2 diabetes: From cardiovascular outcome trials, with hope. Diabetes, Obesity and Metabolism. 2019

13. Yancy CW, Jessup M, Bozkurt B, Butler J, Casey DE, Drazner MH, Fonarow GC, Geraci SA, Horwich T, Januzzi JL, Johnson MR. 2013 ACCF/AHA guideline for the management of heart failure: executive summary: a report of the American College of Cardiology Foundation/American Heart Association Task Force on practice guidelines. Journal of the American College of Cardiology. 2013 Oct 15;62(16):1495-539.

14. Luttik ML, Jaarsma T, van Geel PP, Brons M, Hillege HL, Hoes AW, de Jong R, Linssen G, Lok DJ, Berge M, van Veldhuisen DJ. Long-term follow-up in optimally treated and stable heart failure patients: primary care vs. heart failure clinic. Results of the $\mathrm{COACH}-2$ study. European journal of heart failure. 2014 Nov;16(11):1241-8.

\section{LEARNING POINTS}

- It is imperative that patients with heart failure understand their condition and are involved in their management decisions; lifestyle interventions can improve patients' quality of life and prevent exacerbations.

- Given the complexity of the HF syndrome and its co-morbidities, close collaboration between hospital and primary care practitioners is crucial in order to provide optimal, integrated care and improved outcomes.

- Recent trials (EMPA-REG OUTCOME and the DECLARE TIMI-58) suggest that both empagliflozin and dapagliflozin, which are inhibitors of the sodium glucose co-transporter (SGLT-2) in the kidney, had beneficial prognostic cardiovascular outcomes when added to metformin compared to placebo in patients with cardiovascular risk factors and / or disease.

- Heart failure is increasingly common. Timely and accurate diagnosis is important since treatments can improve symptoms and improve prognosis.

- There are parallels with the diagnostic evaluation of heart failure with both preserved and reduced ejection fraction. Management approached overlap but the syndromes also require distinct treatment strategies.

- Primary care has a crucial responsibility to oversee the health status of a heart failure patient and associated co-morbid conditions using evidence-based pharmacotherapy and lifestyle interventions. 